\title{
Study of a Flexible Low Profile Tunable Dipole Antenna Using Barium Strontium Titanate Varactors
}

\author{
David Cure ${ }^{1}$, Thomas Weller ${ }^{2}$, Félix A. Miranda ${ }^{3}$ \\ 1 (IEEE Member): Research Department, Kymeta Corp., Redmond, WA 98052 USA, dcure@kymetacorp.com \\ 2 (IEEE Senior Member): E.E Department, University of South Florida, Tampa, FL 33620 USA, weller@usf.edu \\ ${ }^{3}$ (IEEE Fellow): Antenna and Optical Systems Branch, NASA John H. Glenn Research Center, Cleveland, OH 44135 USA, \\ felix.a.miranda@nasa.gov
}

\begin{abstract}
In this paper a flexible low profile dipole antenna using a frequency selective surface (FSS) with interdigital barium strontium titanate (BST) varactor-tuned unit cells is presented. The varactor chips were placed only along one dimension of the FSS to avoid the use of vias and simplify the DC bias network. The antenna uses overlapping metallic plates that resemble fish scales as a ground plane to improve the flexibility of the multi-material stack structure. The measured data of the antenna demonstrate tunability from $2.42 \mathrm{GHz}$ to $2.66 \mathrm{GHz}$ and $1.3 \mathrm{~dB}$ gain drop when using overlapping metallic plates instead of continuous ground plane. The total antenna thickness is approximately lambda/24.
\end{abstract}

Index Terms-Flexible antennas, varactor-tuned high impedance surfaces, chip capacitors, BST, Young's module.

\section{INTRODUCTION}

In recent years interest in the development of flexible antennas for portable applications has increased. Their characteristics such as the ability to conform to a surface and low weight make these types of antenna desirable. In this paper a study of a flexible low profile bowtie dipole antenna backed with a barium strontium titanate (BST) varactor-loaded frequency selective surface (FSS) is presented (Fig. 1). The antenna is fed with a microstrip-to-coplanar strip balun and uses two $2.4 \mathrm{~mm}$-thick substrate layers, resulting in a total antenna thickness of $\sim \lambda / 24$ at the operational central frequency of $2.4 \mathrm{GHz}$. The antenna uses overlapping metallic plates to improve the flexibility of the structure.

Typically microwave antennas are made by assembling multiple layers of different materials. Some of these layers are made of metal; generally the backside of the antenna is a metal ground plane and the top side is a metal radiating element. Sandwiched between the metal layers is some kind of non-conducting, insulating material (the substrate). Previous researchers have developed flexible antennas by reducing the thickness of a single substrate layer or by using only one metal layer [1]-[4]. However, these antennas are narrowband and do not meet the requirements of the intended application. In most cases, the antenna performs better as the thickness of the insulating material increases [5]. This is especially true for low profile antennas where the electrical performance (i.e., matching, gain, efficiency, bandwidth, etc.) improves as the antenna thickness increases. On the other

This work was supported by NASA Glenn Research Center's Graduate Student Researcher Program (Grant \# NNX10AL41H), the NACME Alfred P. Sloan Fellowship and the National Science Foundation (grant \#ECS0901779). hand from a mechanical standpoint, flexibility improves with thickness reduction. Stiffness increases with the cube of substrate thickness and stress increases linearly with thickness limiting the amount of deflection before the antenna permanently deforms or breaks [6]. Thus, there is a conflict between improving the antenna performance by increasing the substrate thickness and improving its flexibility by decreasing the thickness.

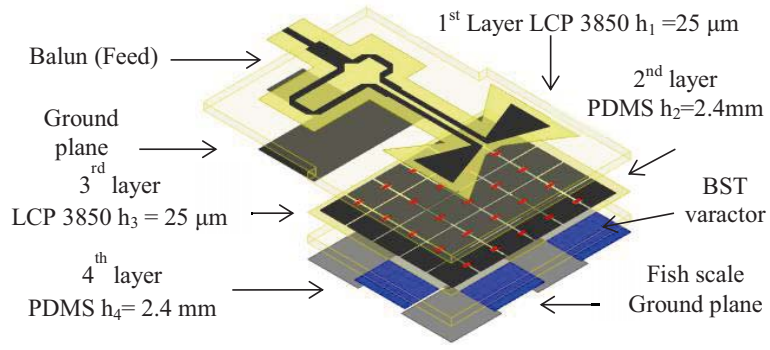

Fig. 1. Schematic of bowtie dipole antenna illustrating the multi-layer substrate with feed layer, tunable FSS layer, and bowtie dipole

This paper proposes increasing the flexibility of a multilayer antenna structure by using overlapping metal plates (fish-scale) which dramatically reduce the rigidity. The antenna design builds upon prior related work on rigid substrates that used a square patch FSS loaded with varactors [7], [8]. To the best of the authors' knowledge this is the first study of a flexible BST varactor-based FSS for low profile antenna applications. All simulation results shown herein were obtained using Ansoft's High Frequency Structure Simulator (HFSS) software.

\section{MECHANICAL AND ELECTRICAL CONSIDERATIONS}

One of the biggest mechanical challenges to address in a multi-material stack structure is how to achieve flexibility. The stiffness of a composite beam is directly proportional to the cube of the thickness and the maximum deformation is experienced by those materials that are farthest from the neutral axis $(O)$. For an antenna, the need to achieve efficient and uni-directional radiation compels the use of a ground plane far from the bending neutral axis $(O)$ which increases the rigidity of the stack. Three different scenarios are 
depicted in Fig. 2; a polymer using only one copper layer (Case I), two copper layers (Case II), and a multi-material stack (Case III). The normalized rigidity was calculated for each case and is shown in Table I. For Case I, the normalized rigidity of a board with polymer height $\left(\mathrm{t}_{\mathrm{PDMS}}\right)$ of $1.25 \mathrm{~mm}$ and one copper layer of thickness $\left(\mathrm{t}_{\mathrm{Cu}}\right) 0.25 \mu \mathrm{m}$ is 1 . If the polymer thickness doubles, then rigidity increases by a factor of 4.3. In this case, the neutral axis does not pass through the centroid of the composite material and it lies closer to the copper layer, reducing the deformation of the latter. However, when a flexible material is sandwiched between two metal layers (Case II), the rigidity is increased by over three orders of magnitude in the examples in Table I. The metal layers will experience more plastic deformation than the polymer as they have a higher modulus of elasticity and are farther from the neutral axis (Fig 2f). For a multi-material stack structure (Case III), the rigidity of the board is increased by factor of 8,300 and 30,000 with respect to case I when $\mathrm{t}_{\mathrm{PDMS}}$ is $1.27 \mathrm{~mm}$ and $2.5 \mathrm{~mm}$, respectively.

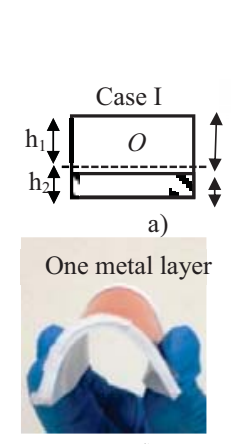

d)

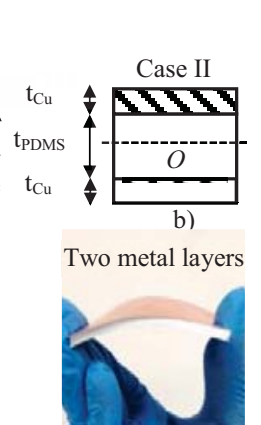

e)

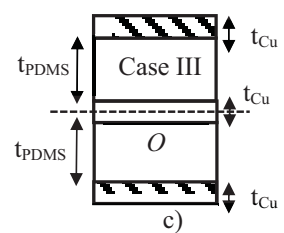

c)

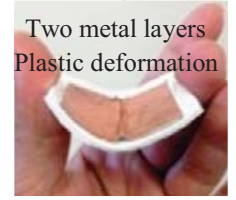

f)
Fig. 2. (a) Rectangular cross section of PDMS based substrate with one metal layer; (b) rectangular cross section of PDMS based substrate sandwiched between two metal layers; (c) rectangular cross section multi-material stack structure, (d) Case I bent forming a negative curvature; (e) Case II bent forming a negative curvature; (f) case II showing plastic deformation of the metal layer.

TABLE I. NORMALIZED FLEXURAL RIGIDITY FOR DIFFERENT SCENARIOS

\begin{tabular}{|c|c|c|c|}
\hline Polymer height & $\begin{array}{c}\frac{\text { Case I }}{\text { One layer }} \\
\text { of copper }\end{array}$ & $\begin{array}{c}\frac{\text { Case II }}{\text { Two layers of }} \\
\text { copper }\end{array}$ & $\begin{array}{c}\frac{\text { Case III }}{\text { Multi-material }} \\
\text { stack }\end{array}$ \\
\hline Rigidity $(1.25 \mathrm{~mm})$ & $1^{*}$ & 1200 & 8300 \\
\hline Rigidity $(2.5 \mathrm{~mm})$ & 4.3 & 8100 & 30000 \\
\hline \multicolumn{3}{|c|}{ * Less rigid case (normalized values) }
\end{tabular}

Another challenge of this design is reducing the losses caused by the series resistance of the BST varactors while using a relatively thin substrate. Full wave analysis of the unit cells using Ansoft HFSS predicts that the series resistance of a varactor has less impact on the antenna performance as the substrate thickness is increased. However, increasing the substrate thickness also increases the rigidity as explained before.

\section{ANTENNA DESIGN, FABRICATION AND RESUlts}

The flexible material selected for the antenna fabrication was polydimethylsiloxane (PDMS) mixed with ceramic loading to achieve miniaturization. The PDMS type selected was Sylgard 184 from Dow Corning which has been widely used for microwave applications [3], [9]-[10]. The ceramic powder used for loading the PDMS was the ultra-low fire UFL990 from Ferro Corp, which is a high dielectric constant ( 90), small particle size $(0.4 \mu \mathrm{m})$ and low loss material. Prior to implementing the multi-layer antenna design the high frequency electrical properties of the materials were determined using Agilent's 85070D dielectric probe kit. Fig. $3 \mathrm{a}$ shows the dielectric constant and electric loss tangent for different volume ratios in a frequency range from $500 \mathrm{MHz}$ to $3 \mathrm{GHz}$. The frequency of operation of the dipole antenna was chosen to be around $\sim 2.4 \mathrm{GHz}$ to be consistent with previous works and to facilitate in-house fabrication (Fig. 3b). The dipole was printed on liquid crystal polymer (LCP). The LCP type used in this work was ULTRALAM 3850 which has a low dielectric constant and low dielectric loss $\left(\varepsilon_{\mathrm{r}}=2.9\right.$ and tan $\delta$ $=0.0025)$. The LCP layer thickness is $25 \mu \mathrm{m}$ with double-side copper cladding of $9 \mu \mathrm{m}$ on which the radiator and the ground plane were patterned using photolithography [11]. The PDMS has an average thickness of $2.5 \mathrm{~mm}$. The LCP and (blended) PDMS were bonded together using SU8-5 photoresist as an intermediate layer [5], [12]-[13]. The SU8-5 was spun onto the LCP at $2000 \mathrm{rpm}(\sim 7 \mu \mathrm{m}$ thickness), then exposed and developed following manufacturer specifications. The SU8 layers were patterned into a square grid (segmented) to increase their flexibility Fig. 3c. The SU8-5 was then treated with (3-Aminopropyl)triethoxysilane (APTES) as explained in [14]. Separately, the PDMS substrate was exposed to oxygen plasma at $10 \mathrm{~W}, 50$ standard cubic centimeters per minute (sccm) for 30 seconds. The LCP and PDMS were aligned with respect to each other and pressed together in a vacuum oven at $70^{\circ} \mathrm{C}$ for 3 minutes to create a permanent bond between the two materials.

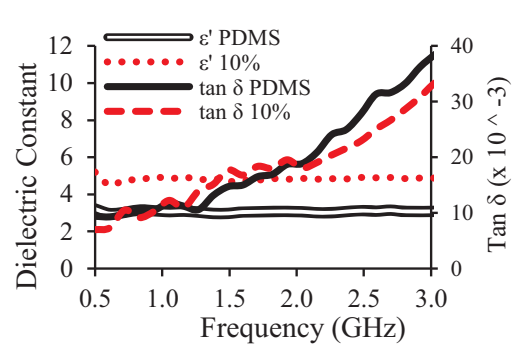

a)

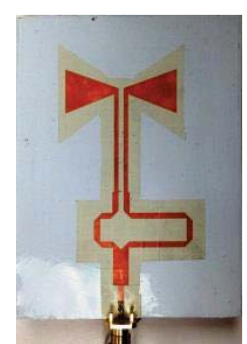

b)

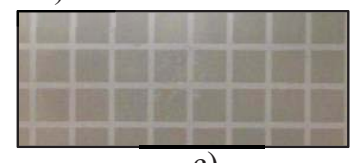

c)

Fig. 3. a) Dielectric constant and loss tangent for the PDMS-ceramic samples at different volume ratios, b) Flexible bowtie antenna and c) SU8 Patterned on the LCP.

The FSS and antenna substrates were cured at ambient temperature over a leveled optical table to maintain a uniform 
height and to avoid an increment on the Young's modulus of the material [14]. The maximum variation allowed for the substrate height was $\sim \pm 0.1 \mathrm{~mm}$ to minimize possible changes in the frequency response. The fabrication of these flexible antenna substrates as well as the LCP/PDMS bonding process is explained in detail in [14].

Simulations of the magnitude of the reflection coefficient ( $\Gamma$ ) of a unit cell using different substrate losses and two different substrate heights are depicted in Fig. 4. The results show that the magnitude of reflection coefficient $(\Gamma)$ decreases with increasing substrate loss tangent and $(\Gamma)$ is particularly degraded when the FSS substrate is thinner. Also, as it was previously discussed in section II, increasing the substrate thickness increases the rigidity. Fig. 5 shows the effect of the equivalent series resistance of the varactor on the magnitude of the reflection coefficient for different substrate thicknesses.
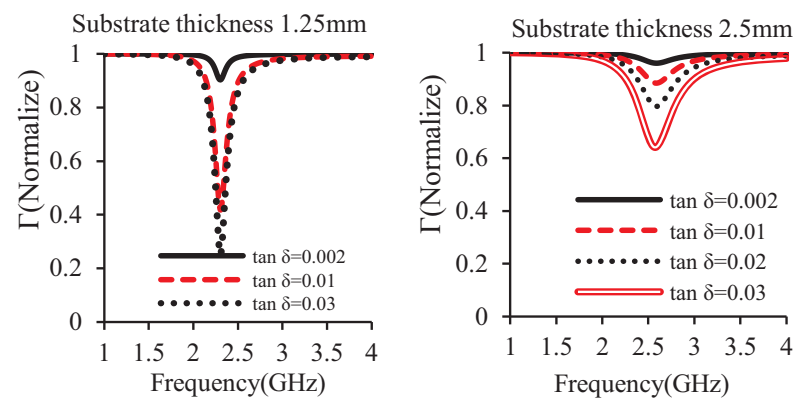

Fig. 4. Impact of the substrate losses and thickness on the reflection coefficient magnitude of the tunable FSS.
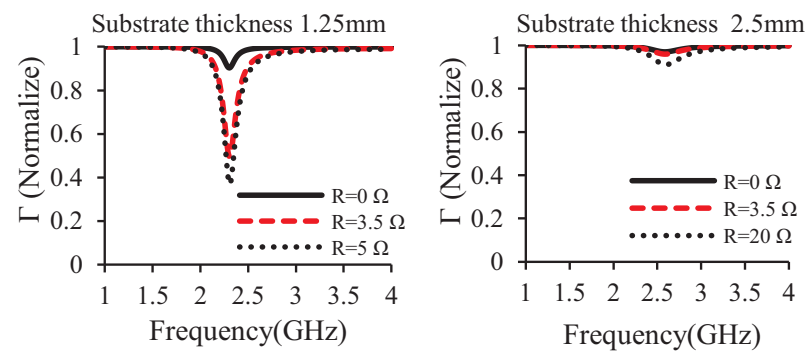

Fig. 5. Impact of the substrate thickness and varactor losses on the reflection coefficient magnitude of the tunable FSS. Substrate $\tan \delta=0.002$.

To validate the impact on the antenna gain due to variations on the FSS height, three FSS's with different substrate heights were fabricated with SMD chip capacitors (equivalent series resistance, $\mathrm{ESR}=\sim 0.5 \Omega$ ). All three FSS's consisted of 30 unit cells and 25 chip capacitors and they were designed to operate at $\sim 2.4 \mathrm{GHz}$, but built with substrates thicknesses of $2.0 \mathrm{~mm}$, $2.3 \mathrm{~mm}$ and $2.5 \mathrm{~mm}$ and capacitance values of $1.2 \mathrm{pF}, 0.7 \mathrm{pF}$ and $0.5 \mathrm{pF}$, respectively. $\mathrm{S}_{11}$ of the antenna backed with the three capacitive loaded FSS's are shown in Fig. 6. The antenna backed with the $2.5 \mathrm{~mm}$ height FSS had the highest gain $(0.8 \mathrm{~dB})$ and better impedance matching than the others. The gain of the $2.3 \mathrm{~mm}$ and $2.0 \mathrm{~mm}$ designs was $0.6 \mathrm{~dB}$ and $3.5 \mathrm{~dB}$ lower, respectively, with respect to the $2.5 \mathrm{~mm}$ height. These results are in agreement with the unit cell simulations in Fig. 5.
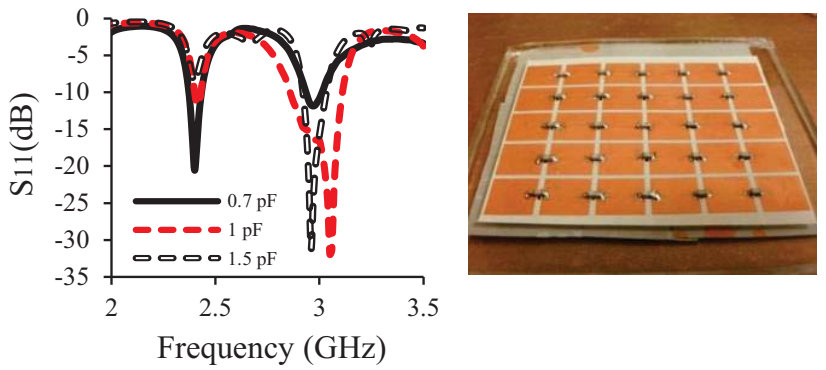

Fig. 6. Measured and simulated $\mathrm{S}_{11}$ for different capacitance values (left) and capacitive loaded FSS.

To design the tunable FSS, simulations of the phase and the magnitude of the reflection coefficient $(\Gamma)$ of a unit cell were performed. The capacitance was varied from $1 \mathrm{pF}$ to $1.5 \mathrm{pF}$ to correspond with the approximate measured tunable range of the BST varactors and the FSS thickness of $\sim 2.4 \mathrm{~mm}$ was assumed. The results in Fig. 7 predict a tunable bandwidth of $400 \mathrm{MHz}$, from $2.2 \mathrm{GHz}$ to $2.6 \mathrm{GHz}$, when the criterion of $0 \pm$ $45^{\circ}$ phase shift is used. These results take into account an extracted varactor series resistance of $3.5 \mathrm{ohms}$ and a substrate loss tangent of 0.02 [7].

The tunable FSS was fabricated using a $\sim 2.4$ mm-thick $10 \%$ volume blended PDMS ceramic substrate, with a dielectric constant of $\sim 5$. The number of devices and unit cell sizes remained the same. The FSS has a planar size of $64 \times 65$ $\mathrm{mm}^{2}$, including the bias network. This bias network is distributed in 5 columns, each containing seven BST chips in series, and 6 rows with $1 \mathrm{k} \Omega$ resistors in series. The antenna has six independent DC voltage lines, on which the odd lines $\left(\mathrm{V}_{1}, \mathrm{~V}_{3}, \mathrm{~V}_{5}\right)$ are biased at the same voltage but opposite polarity with respect to the even lines $\left(\mathrm{V}_{2}, \mathrm{~V}_{4}, \mathrm{~V}_{6}\right)$ to create a virtual ground between them (Table II). This bias network takes advantage of the symmetric behavior along the zero-bias voltage axis in the $\mathrm{C}-\mathrm{V}$ curve and reduces the maximum required voltage [7]. Similar to [8], the varactors were placed in the direction parallel to the main axis of the bowtie dipole to achieve higher tunability. A $1 \mathrm{k} \Omega$ resistor was used at the ends of each row to block RF leakage onto the bias lines. The FSS's ground plane has overlapping metallic plates instead of a continuous metal layer to improve flexibility. Fig. 8 shows the fish scale ground plane and the fabricated tunable FSS.

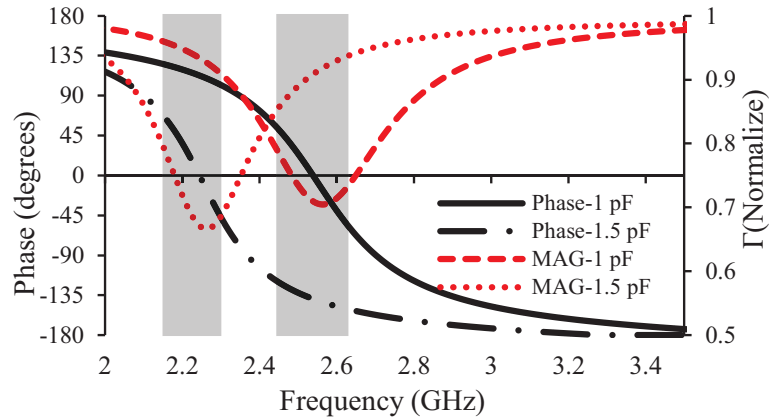

Fig. 7. Simulated reflection coefficient phase and magnitude of the flexible tunable FSS. 
TABLE II. FSS BIAS CONFIGURATIONS

\begin{tabular}{|c|c|c|c|c|c|c|}
\hline Config. & $\boldsymbol{V}_{\boldsymbol{1}}$ & $\boldsymbol{V}_{\mathbf{2}}$ & $\boldsymbol{V}_{\mathbf{3}}$ & $\boldsymbol{V}_{\mathbf{4}}$ & $\boldsymbol{V}_{\mathbf{5}}$ & $\boldsymbol{V}_{\mathbf{6}}$ \\
\hline $\mathrm{A}$ & $0 \mathrm{~V}$ & $0 \mathrm{~V}$ & $0 \mathrm{~V}$ & $0 \mathrm{~V}$ & $0 \mathrm{~V}$ & $0 \mathrm{~V}$ \\
\hline $\mathrm{B}$ & $30 \mathrm{~V}$ & $-30 \mathrm{~V}$ & $30 \mathrm{~V}$ & $-30 \mathrm{~V}$ & $30 \mathrm{~V}$ & $-30 \mathrm{~V}$ \\
\hline $\mathrm{C}$ & $50 \mathrm{~V}$ & $-50 \mathrm{~V}$ & $50 \mathrm{~V}$ & $-50 \mathrm{~V}$ & $50 \mathrm{~V}$ & $-50 \mathrm{~V}$ \\
\hline
\end{tabular}

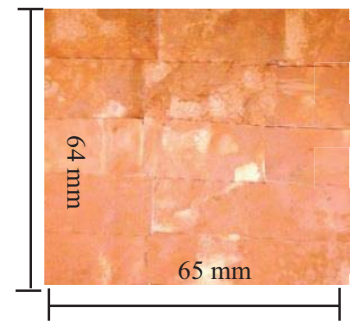

Fig. 8. Ground plane with overlapping metallic layers (left) and tunable FSS with bias network (right)

Measured $\mathrm{S}_{11}$ data for the antenna when applying a common bias voltage of 0 and $\pm 50 \mathrm{~V}$ to the DC bias ports are shown in Fig. 9. Using the $10 \mathrm{~dB}$ return loss criterion, there is a $280 \mathrm{MHz}$ span between the low end of the response with 0 $\mathrm{V}$ and the high end of the response using $50 \mathrm{~V}$ (shaded region in Fig.9). Two additional resonances appear at $\sim 2.1 \mathrm{GHz}$ and $3 \mathrm{GHz}$ when the input bias is $0 \mathrm{~V}$ and then these are shifted up $\sim 200 \mathrm{MHz}$ when the voltage is $50 \mathrm{~V}$ as consequence of TE surface wave propagation. The resonant frequencies of these modes can be calculated using the cavity model analysis explained in detail in [15]; this analysis predicts a TE resonance at $\sim 2.07 \mathrm{GHz}$ and $3.1 \mathrm{GHz}$ for a FSS structure composed of five unit cells with periodicity of $9.9 \mathrm{~mm}$.

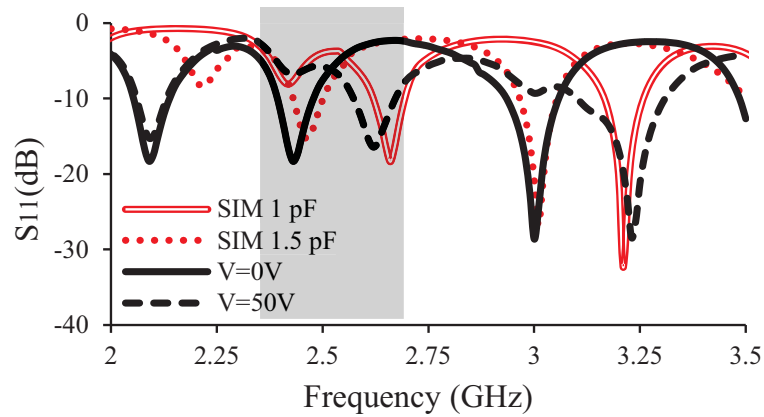

Fig. 9. Measured and simulated $S_{11}$ when $0 \mathrm{~V}$ and $\pm 50 \mathrm{~V}$ is applied to all bias ports.

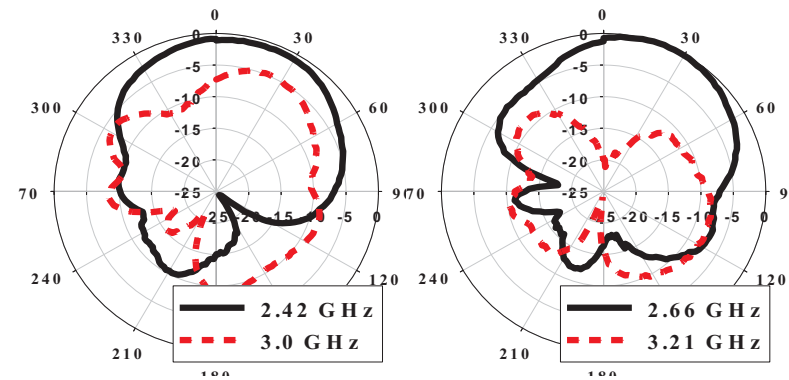

Fig. 10. Measured E-plane radiation patterns for the antenna with bias voltage of $0 \mathrm{~V}$ (left) and $\pm 50 \mathrm{~V}$ (left) at different frequencies.
The E-plane radiation patterns of the antenna for different bias voltages are shown in Fig. 10. The radiation patterns of the antenna with bias voltage of $0 \mathrm{~V}$ and $\pm 50 \mathrm{~V}$ demonstrate cancellation of back radiation at $2.42 \mathrm{GHz}$ and $2.66 \mathrm{GHz}$, which is within to the operational frequency of the tunable antenna shown in Fig.9. The patterns are rotated $\sim 25^{\circ}$ due to the presence of surface waves. The effects of surface waves are observed in the radiation pattern due to the absence of vias in the high impedance surface. Fig. 10 also depicts the patterns at $3.0 \mathrm{GHz}$ and $3.21 \mathrm{GHz}$ which suggest the presence of leaky waves. The dispersion diagram of the 6 cascaded unit cells for different capacitance values was simulated using one dimensional (1D) simulation in HFSS. The Bloch dispersion diagram was calculated using the scattering parameters taking into account the number of cells along the direction of the electric field of the dipole (linearly polarized) which is where major excitation of surface waves is produced. The dispersion diagram shows backward/forward leaky waves at $\sim 3 \mathrm{GHz}$ for a capacitance value of $1.5 \mathrm{pF}$ and at $\sim 3.2 \mathrm{GHz}$ for $1 \mathrm{pF}$. The leaky waves are supported in the fast wave region indicated to the left side of the light line shown in Fig. 11a. The magnitude of the tangential electric fields of 6 cascaded unit cells for $\mathrm{C}=1.0 \mathrm{pF}$ at $3.2 \mathrm{GHz}$ (leakage) and at $2.3 \mathrm{GHz}$ (no leakage) are depicted in Fig. $11 \mathrm{~b}$.

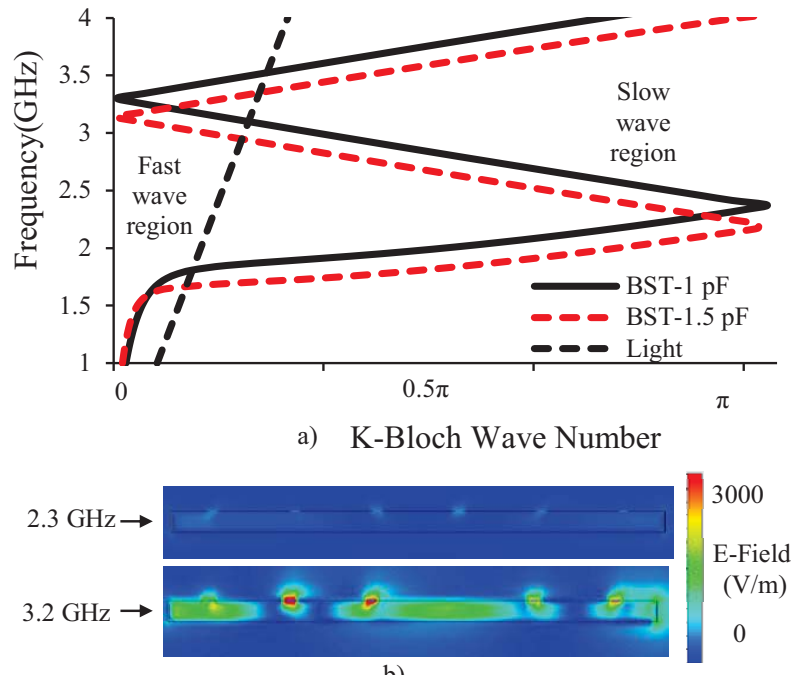

b)

Fig. 11. (a) Dispersion diagram obtained by cascading 6 unit cellls with periodicity of $9.9 \mathrm{~mm}$ for $\mathrm{C}=1 \mathrm{pF}$ and $\mathrm{C}=1.5 \mathrm{pF}$. (b) Magnitude of the tangential electric fields along the 8 unit cells for $\mathrm{C}=1 \mathrm{pF}$ at $3.2 \mathrm{GHz}$ (leakage) and at $2.3 \mathrm{GHz}$ (no leakage).

The gains of the antenna backed with an FSS using a continuous and a fish scale ground plane were compared to each other. The continuous ground plane case was obtained by covering the fish scales with adhesive copper tape. The measured gain for the fish scale case was $-0.86 \mathrm{dBi}$ at 2.4 $\mathrm{GHz}$ for a $0 \mathrm{~V}$ input bias and for the continuous case the gain was $0.4 \mathrm{dBi}$. This represents a $\sim 1.3 \mathrm{~dB}$ gain reduction when using the fish-scale metal layer instead of a continuous ground. The low gain in both cases may be attributed to the 
material losses and variations on the FSS height. The simulated antenna gain at broadside obtained with Ansoft HFSS using a continuous ground plane is approximately 1.6 $\mathrm{dBi}$ at $2.4 \mathrm{GHz}$; however the 0.04 loss tangent of the SU8-5 bond layer and possible variations of the substrate height were not included in the model to reduce the computational requirements.

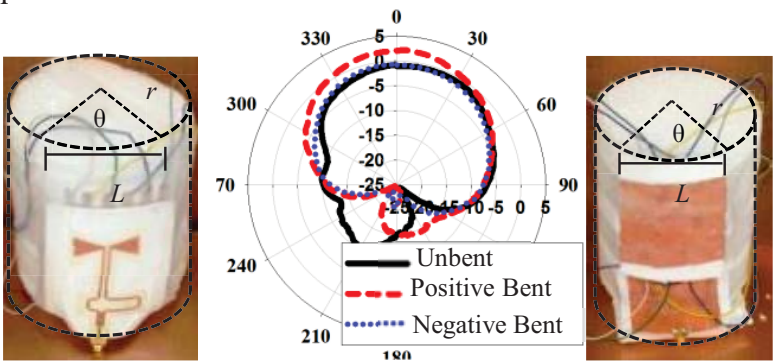

Fig. 12. Measured E-plane radiation patterns for the antenna with bias voltage of $0 \mathrm{~V}$ bent with negative (left) and positive (right) curvature for $\theta=77^{\circ}$.

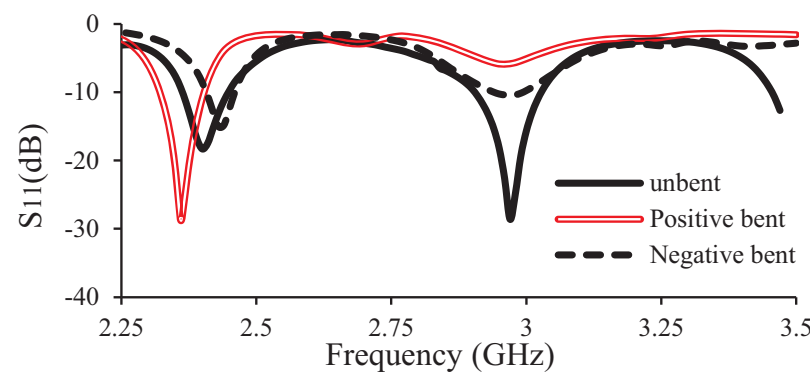

Fig. 13. Measured $\mathrm{S}_{11}$ of the bowtie dipole antenna backed with an FSS unbent, bent with positive curvature and negative curvature for $\theta=77^{\circ}$.

A styrofoam cylinder of $50 \mathrm{~mm}$ radius $r$ was used to perform the bending tests. The angle of curvature $\theta$ was determined using the formula which defines the central angle whose vertex is the center of a circle $(L=64 \mathrm{~mm})$. Fig. 12 and Fig. 13 show the radiation pattern and $\mathrm{S}_{11}$ data when the antenna is unbent and bent (positive and negative). The results show that when the antenna is bent in positive curvature the frequency shifts up $40 \mathrm{MHz}(1.6 \%)$ and the gain at $2.36 \mathrm{GHz}$ increases by $3.1 \mathrm{~dB}$ for an angle of $77^{\circ}$ for $\mathrm{V}=0 \mathrm{~V}$ with respect the unbent case. This gain increase is realized because there is less back radiation as the angle increases and the ground plane curves toward the dipole direction. For the negative bending case the frequency shifts up $20 \mathrm{MHz}(0.86 \%)$ and the gain mantains similar values as the unbent antenna $(\sim-0.7 \mathrm{~dB})$, but there is less back radiation as consequence of the curvature of the ground plane.

\section{CONCLUSION}

The fish-scale ground plane was demonstrated specifically for flexible microwave antennas. The approach represents a significant advance in the combined thickness and flexibility that can be achieved, especially when using relatively thick substrates which are important for optimum antenna performance. An increase in gain and further reduction of back radiation were observed when the antenna was bent in a positive radius of curvature. The demonstrations that have been done in this paper using a hybrid manufacturing approach could be transitioned to thin-film microelectronics.

\section{ACKNOWLEDGMENT}

The authors thank Rogers Corporation for donating the LCP substrates used in this work. The authors also thank Maria F. Córdoba from USF for her support during antenna measurements and fabrication.

\section{REFERENCES}

[1] A. Tronquo, H. Rogier, and C. Hertleer, "Robust planar textile antenna for wireless body LANs operating in $2.45 \mathrm{GHZ}$ ISM band," Electronics Letter, vol. 42, no.3, pp. 142-143, February 2006.

[2] X. Zhang, A. Zhao, and J. Wang, "Research on the characteristics of flexible antennas for general applications," in Microw. and Millimeter Wave Technol. Int. Conf., Apr. 21-24, 2008, vol. 4, pp. 1814-1817

[3] D.E. Anagnostou, A.A. Gheethan, A.K. Amert, and K.W. Whites, A direct-write printed antenna on paper-based organic substrate for flexible displays and WLAN applications, IEEE J Display Technol 6 ( 2010), 558-564

[4] A. Tronquo, H. Rogier, and C. Hertleer, "Robust planar textile antenna for wireless body LANs operating in $2.45 \mathrm{GHZ}$ ISM band, " Electronics Letter, vol. 42, no.3, pp. 142-143, February 2006

[5] C.-P. Lin, C.-H. Chang, Y.T. Cheng, C.F. Jou, "Development of a flexible SU-8/PDMS-based antenna", IEEE Antennas Wirel. Propag. Lett., 10 1108-1111 (2011).

[6] J. M. Gere, S. P. Timoshenko "Mechanics of Materials", 4th ed, PWS Pub. Co, MA, 1998, pp. 314-317.

[7] D. Cure, T. Weller, T.Price, F. Van keuls and F.A. Miranda "Low Profile Tunable Dipole Antenna Using Barium Strontium Titanate Varactors," to be published at Antennas and Propagation, IEEE Transactions on, Vol. 62 No. 03, March 2014.

[8] D. Cure, T. Weller and F.A. Miranda "Study on a Low Profile $2.4 \mathrm{GHz}$ Planar Dipole Antenna Using a 1-D Varactor-Tuned High Impedance Surface," Antennas and Propagation, IEEE Transactions on, vol.61, no.2, pp.1, February 2013

[9] S. Koulouridis, G. Kiziltas, Y. Zhou, J L. Hansford, and D. J. Volakis, "Polymer-Ceramic Composites for Microwave Applications: Fabrication and Performance Assessment," IEEE Trans. Microw. Theory Tech., 54 4202-4208 (2006).

[10] C. Morales, "Magneto-Dielectric Polymer Nanocomposite Engineered Substrate for RF and Microwave Antennas" Ph.D. Thesis, Dept. Elect. University of South Florida, Tampa, FL, 2011.

[11] A Volkov, V Pavelyev, O Moiseev, V Eropolov, B Volodkin and K Tukmakov, "Thin copper film for plasma etching of quartz," Optical Memory and Neural Networks (Information Optics), 18(1):40-43 (2009).

[12] S.L. Peterson, A. McDonald, P.L Gourley, D.Y. Sasaki, "Poly(dimethylsiloxane) thin films as biocompatible coatings for microfluidic devices: Cell culture and flow studies with glial cells Biomed," Mater. Res. A 2005, 72A, 10-18.

[13] S. Talaei, O, Frey, P.D. van der Wal, N.F. de Rooij and M. KoudelkaHep, "Hybrid microfluidic cartridge formed by irreversible bonding of SU-8 and PDMS for multi-layer flow applications", Procedia Chemistry 1, 381-384 (2009).

[14] D. Cure, "Reconfigurable Low Profile Antennas Using Tunable High Impedance Surfaces" Ph.D. Thesis, Dept. Elect. University of South Florida, Tampa, FL, 2013

[15] F. Costa, O. Luukkonen, C.R. Simovski, A. Monorchio, S.A. Tetryakov and P. de Maagt, "TE Surface Wave Resonances on High-Impedance Surface Based Antennas: Analysis and Modeling," IEEE Trans Antennas Propagat., vol. 59, no. 10, pp. 3588-3596, Oct. 2011. 